\title{
A Novel Gas Turbine Engine Health Status Estimation Method Using Quantum-Behaved Particle Swarm Optimization
}

\author{
Xinyi Yang, ${ }^{1}$ Wei Shen, ${ }^{1}$ Shan Pang, ${ }^{2}$ Benwei Li, ${ }^{1}$ Keyi Jiang, ${ }^{1}$ and Yonghua Wang ${ }^{1}$ \\ ${ }^{1}$ Department of Aerocraft Engineering, Naval Aeronautical and Astronautical University, Yantai 264001, China \\ ${ }^{2}$ College of Information and Electrical Engineering, Ludong University, Yantai 264025, China \\ Correspondence should be addressed to Xinyi Yang; jimyang2008@163.com
}

Received 25 April 2014; Revised 6 July 2014; Accepted 7 July 2014; Published 6 August 2014

Academic Editor: George Tsiatas

Copyright (c) 2014 Xinyi Yang et al. This is an open access article distributed under the Creative Commons Attribution License, which permits unrestricted use, distribution, and reproduction in any medium, provided the original work is properly cited.

\begin{abstract}
Accurate gas turbine engine health status estimation is very important for engine applications and aircraft flight safety. Due to the fact that there are many to-be-estimated parameters, engine health status estimation is a very difficult optimization problem. Traditional gas path analysis (GPA) methods are based on the linearized thermodynamic engine performance model, and the estimation accuracy is not satisfactory on conditions that the nonlinearity of the engine model is significant. To solve this problem, a novel gas turbine engine health status estimation method has been developed. The method estimates degraded engine component parameters using quantum-behaved particle swarm optimization (QPSO) algorithm. And the engine health indices are calculated using these estimated component parameters. The new method was applied to turbine fan engine health status estimation and is compared with the other three representative methods. Results show that although the developed method is slower in computation speed than GPA methods it succeeds in estimating engine health status with the highest accuracy in all test cases and is proven to be a very suitable tool for off-line engine health status estimation.
\end{abstract}

\section{Introduction}

The gas turbine engine is an aircraft power plant and is very important for flight safety. During its operation life, the gas turbine engine is susceptible to a lot of physical problems, including corrosion, erosion, fouling, foreign object damages, excessive tip clearance, and warped stator or rotor blades [1]. As a result, the engine performance will deteriorate gradually. Therefore, it is necessary to estimate engine health status after a period of operation time for aircraft flight safety. Engine performance deterioration can be represented by engine health status indices of engine components such as compressor and turbine. But these parameters are not directly measured and are very difficult to obtain.

Some studies have been done in the field of gas turbine engine performance and health status analysis in recent years. Louis Urban [1] first presented a gas path analysis (GPA) method which interrelates measurable engine parameters and engine health parameters using fault coefficient matrix and applied the method to turbine engine condition monitoring. Roth et al. [2] developed an optimization technique for a turbofan engine cycle model matching process and a minimum variance estimator algorithm [3] for engine performance matching to test data. Li and Pilidis applied genetic algorithm adaptation method [4] to gas turbine design-point performance adaptation and compared it with influence coefficient matrix- (ICM-) based method. In [5], Li developed an adaptive gas path analysis and applied it to gas turbine performance and health status estimation. Other techniques applied to engine performance and health status analysis include neural networks [6], Bayesian belief networks [7], and fuzzy logic [8,9]. These methods can obtain good estimation results on engine design-point condition. However, they may not provide satisfactory results on engine off-design point conditions.

In essence, gas turbine engine health status estimation is a difficult optimization problem with many health parameters to estimate. As the application of QPSO to engine performance adaptation or health estimation has not been reported in the literature, in this study, a novel gas turbine engine health status estimation method using quantum-behaved 
particle swarm optimization has been developed. The method was applied to the health status estimation of a two-shaft turbine fan engine with afterburner. Then, the comparison with other contemporary methods including adaptive gas path analysis and GA-based methods in different cases was carried out. Results show that the proposed method outperforms the compared methods in several ways and provides satisfactory estimation results on both engine design-point and off-design point conditions. It is proven to be a good tool for off-line gas turbine engine health status estimation.

\section{Methodology for Gas Turbine Engine Health Status Estimation}

2.1. Performance Adaptation Approach. The thermodynamic relationship between gas turbine engine measurement parameters and engine components performance parameters at a certain ambient and operating condition can be represented by the following equation [5]:

$$
\mathbf{z}=h(\mathbf{x})
$$

where $\mathbf{z} \in R^{M}$ refers to the gas path measurement parameter vector, $M$ is the number of measurement parameters, $\mathbf{x} \in R^{N}$ is the component performance parameter vector, $N$ is the number of component performance parameters, and $h(\cdot)$ is a vector valued function representing engine thermodynamic relationship between $\mathbf{x}$ and $\mathbf{z}$.

When an engine is undeteriorated, the performance of the engine is denoted by subscript " 0 ". If the engine operates at a deteriorated condition, the engine performance represented by (1) can be expanded to a Taylor series expansion as the following equation:

$$
\mathbf{z}=\mathbf{z}_{0}+\left.\frac{\partial h(\mathbf{x})}{\partial \mathbf{x}}\right|_{0}\left(\mathbf{x}-\mathbf{x}_{\mathbf{0}}\right)+\mathrm{HOT},
$$

where HOT is the higher order terms of the expansion and can be neglected when the performance deviation is small. Therefore, a linearized gas turbine performance model can be expressed as the following equation:

$$
\Delta z=\mathbf{z}-\mathbf{z}_{0}=\mathbf{H} \Delta \mathbf{x}
$$

The deviation of component performance parameters can be then predicted by inverting the influence coefficient matrix (ICM) $H$ to an adaptation coefficient matrix (ACM) $H^{-1}$, leading to (4) when $M=N$. In some cases, $M$ is less than $N$. In this study, we focus on the condition that $M$ equals $N$ :

$$
\Delta \mathbf{x}=\mathbf{H}^{-1} \Delta \mathbf{z}
$$

Then the deviated engine performance can be estimated with

$$
\mathbf{x}=\mathbf{x}_{\mathbf{0}}+\Delta \mathbf{x}
$$

where $\mathbf{x}_{0}$ is the original engine component performance parameter vector, while $\Delta \mathbf{x}$ is the deviation of component performance parameter vector indicated by the change in gas path measurements $\Delta \mathbf{z}$. The method described above is called linear performance adaptation.

As the engine performance may deviate nonlinearly from its initial baseline condition because of nonlinear thermodynamic behavior of engine performance, the linear performance adaptation may not be able to provide accurate engine health status estimation. Therefore an iterative process is introduced to improve the estimation accuracy. The linear adaptation is applied iteratively until a converged solution is obtained. The above method is called the nonlinear performance adaptation. Theoretically, the nonlinear performance adaptation has the potential to provide better adaptation results than linear performance adaptation.

2.2. Adaptive Linear GPA and Adaptive Nonlinear GPA Methods. Li developed adaptive GPA methods [5] based on linear performance adaptation described in Section 2.1. There are mainly two steps included in the adaptive GPA method: the first step is the estimation of degraded engine performance status by linear or nonlinear performance adaptation, and the second step is the estimation of engine health status at component level.

For adaptive linear GPA method, the higher order terms of (2) are neglected; thus the gas turbine performance model is expressed into the simple form as (3). When performance deviation is small, adaptive linear GPA method is able to provide accurate engine health status estimation. But when the engine works at an operating condition which is far away from the design point, the HOT becomes significant. In that case, adaptive linear GPA method may fail to provide accurate estimation solutions.

At the same time, the influence coefficient matrix $\mathbf{H}$ is obtained from engine model at a certain operating condition and the components performance variations are not considered. Thus $\mathbf{H}$ maintains the same value even the component performances deteriorate from initial healthy state. This may decrease the GPA methods estimation accuracy.

Li also developed an adaptive nonlinear GPA method. It may increase the estimation accuracy by repeating the linear performance adaptation iteratively. But, in essence, the method is still based on the linearized model. When the nonlinearity of the relationship between the component parameters and performance parameters is significant, even adaptive nonlinear GPA may fail to provide solutions with high accuracy.

\section{QPSO and Its Application in Engine Health Status Estimation}

3.1. QPSO and Other Population-Based Optimization Algorithms. The primary object of gas turbine engine health status estimation is to obtain the "real" engine components parameters by some methods. This problem is inherently nondeterministic in nature. At certain engine operating condition, candidate engine components parameters are randomly generated by certain algorithm and then the engine performance parameters can be calculated from an engine model using these candidate parameters. The difference 
between the calculated performance parameters and test data is then evaluated by some criterion (e.g., smaller than $\sigma$ ). The process is repeated until the criterion is satisfied. Therefore gas turbine engine health status estimation can be regarded as an optimization problem fundamentally.

In this problem, the to-be-optimized parameters are deteriorated gas turbine engine components performance parameters. And engine gas path measurement parameters are used as the target parameters. The differences between calculated and real engine gas path measurement parameters are used by some optimization algorithm to estimate engine components performance parameters. These calculated components performance parameters are then used to obtain engine health status indices by comparing with the initial (i.e., undeteriorated) components performance parameters.

In recent years, some population-based random optimization algorithms, such as genetic algorithm (GA) and particle swarm optimization (PSO), have been widely used in solving complex optimization problems. Li and Pilidis have applied GA to engine design-point performance adaptation and compared it with ICM-based approach [4]. Results show that GA-based method is more robust numerically than ICMbased method, but its prediction accuracy is slightly lower.

Unlike GA which was motivated by Darwin's natural evolution theory, PSO was originally proposed by Kennedy and Eberhart as a simulation of bird flock's social behavior and was applied in solving optimization problems in 1995 [10]. Instead of using evolutionary operators such as mutation and manipulating to generate new individuals during evolution, PSO relies on the information exchange between particles. Each particle of the population flies in search space with a certain velocity, which is dynamically adjusted according to global best and personal best solutions. PSO has no evolutionary operators and is simple in use compared with GA. Many researches proved that PSO shows better performance in complex optimization problems [11-13].

However, PSO is not a global optimization algorithm and has premature or local convergence problems, as has been demonstrated by van den Bergh and Engelbrecht [14]. To solve these problems, Sun et al. introduced the quantum theory into PSO algorithm and proposed a quantumbehaved PSO (QPSO) algorithm [15]. Results on some typical benchmark functions show that the QPSO works better than standard PSO and it is theoretically guaranteed to find optimal solution in search space.

In this research QPSO is applied to solve this optimization problem. QPSO is a novel optimization algorithm inspired by the fundamental theory of particle swarm and features of quantum mechanics. It was initially developed to deal with PSO's main limitation of premature convergence. In QPSO, the state of a particle $\mathbf{y}$ is depicted by Schrodinger wave function $\psi(\mathbf{y}, t)$, instead of position and velocity. The dynamic behavior of the particle is widely divergent from classical PSO systems in that the exact values of position and velocity cannot be determined simultaneously. We can only learn the probability of the particle's appearing in a position by probability density function $|\psi(\mathbf{y}, t)|^{2}$. Employing the Monte
Carlo method, for the $i$ th particle $\mathbf{y}_{i}$ from the population, the particle moves according to the following iterative equation:

$$
\begin{aligned}
\mathbf{y}_{i, j}(t+1)= & \mathbf{P}_{i, j}(t)-\beta \cdot\left(\text { BBest }_{j}(t)-\mathbf{y}_{i, j}(t)\right) \\
& \cdot \ln \left(\frac{1}{u_{i, j}(t)}\right) \quad \text { if } k \geq 0.5, \\
\mathbf{y}_{i, j}(t+1)= & \mathbf{P}_{i, j}(t)+\beta \cdot\left(m \text { Best }_{j}(t)-\mathbf{y}_{i, j}(t)\right) \\
& \cdot \ln \left(\frac{1}{u_{i, j}(t)}\right) \quad \text { if } k<0.5,
\end{aligned}
$$

where $\mathbf{y}_{i, j}(t+1)$ is the position of the $i$ th particle with respect to the $j$ th dimension in iteration $t . \mathbf{P}_{i, j}$ is the best position of the $i$ th particle to the $j$ th dimension and is defined as

$$
\begin{gathered}
\mathbf{P}_{i, j}(t)=\varphi_{j}(t) \cdot \text { pBest }_{i, j}(t)+\left(1-\varphi_{j}(t)\right) g \text { Best }_{j}(t), \\
\text { mBest }_{j}(t)=\frac{1}{\mathrm{NP}} \sum_{i=1}^{\mathrm{NP}} \text { pBest }_{i, j}(t),
\end{gathered}
$$

where NP is the number of particles and $p$ Best $_{i}$ represents the best previous position of the ith particle. gBest is the global best position of the particle swarm. mBest is the mean best position defined as the mean of all the best positions of the population, and $k, u$, and $\varphi$ are random numbers distributed uniformly on $[0,1]$, respectively. $\beta$ is called contractionexpansion coefficient. It can be tuned to control the convergence speed of the algorithms. Experiments show that QPSO shows better convergence performance than standard PSO, GA, and some other algorithms in solving typical benchmark optimization problems $[16,17]$.

3.2. Engine Health Status Estimation Using QPSO. When QPSO is applied to engine health status estimation, the optimization procedure can be illustrated as in Figure 1. QPSO first generates a population composed of solutions (particles) $\mathbf{x}, \mathbf{x}=\left\{x_{1}, x_{2}, \ldots, x_{N}\right\}$, where $\mathbf{x}$ denotes a potential solution consisting of the component parameters such as compressor gas flow rate and efficiency.

Then the components characteristic maps are used to obtain corresponding low-pressure compressor pressure ratio $\pi_{\mathrm{cl}}$, high-pressure compressor pressure ratio $\pi_{\mathrm{ch}}$, lowpressure turbine enthalpy drop $\Delta H_{\mathrm{tl}}$, and high-pressure turbine enthalpy drop $\Delta H_{\text {th }}$. The method to obtain $\pi_{\mathrm{cl}}$ according to shaft rotational speed $n_{l}$ and gas flow rate $w_{\mathrm{cl}}$ from lowpressure compressor characteristics map can be illustrated as in Figure 2.

It should be pointed out that when the engine degrades, the shape of the component map should change to reflect the degradation. Thus before obtaining $\pi_{\mathrm{cl}}$, the original component needs to be scaled using scale factor as introduced in [18]. The scale factor for this example is the division of the generated $w_{\mathrm{cl}}$ and engine undeteriorated $w_{\mathrm{cl}, 0}$ at the same operating condition.

Likewise, the turbine enthalpy drop can be obtained by the same way. Then, these parameters and engine condition 


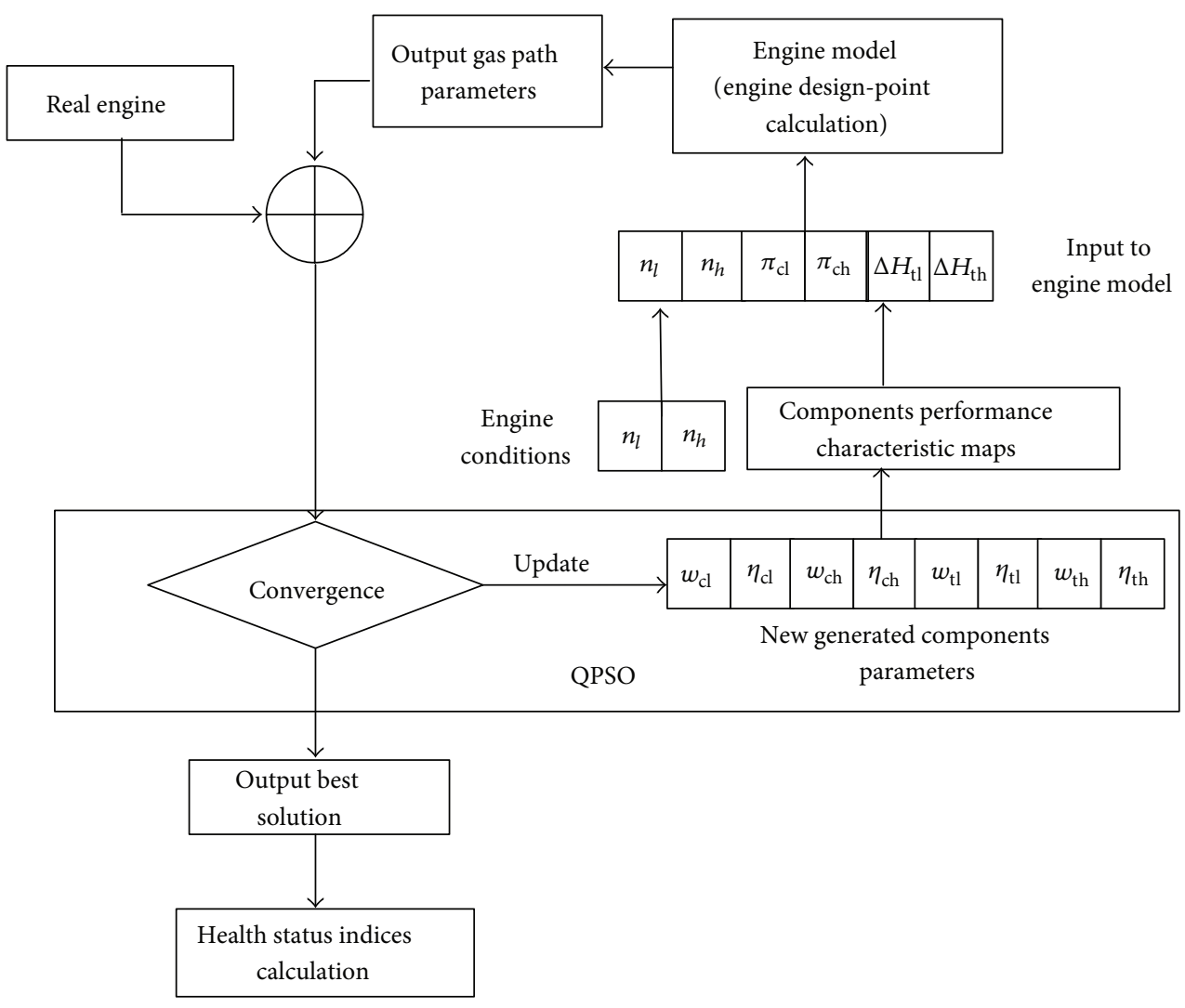

FIGURE 1: Optimization procedure using QPSO.

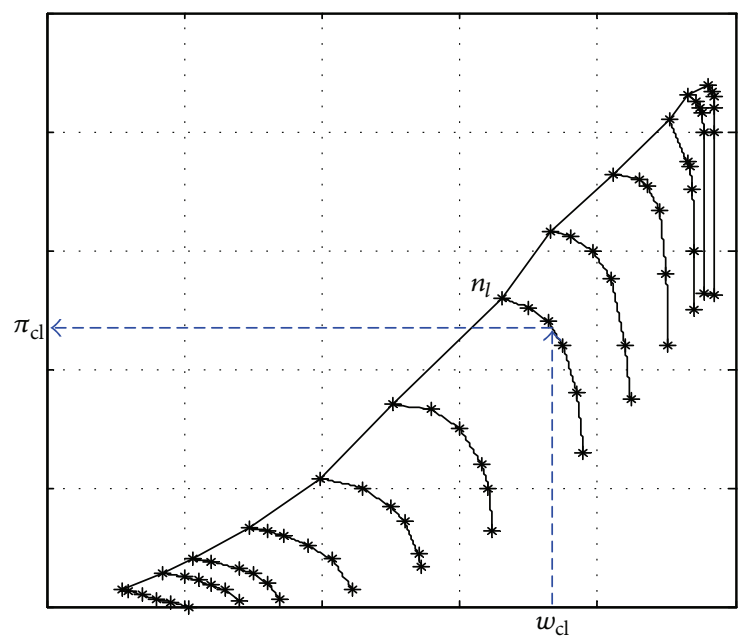

FIGURE 2: Illustration of obtaining compressor pressure ratio $\pi_{\mathrm{cl}}$ from characteristics map.

parameters are input to thermodynamic engine performance model to obtain gas path parameters. The difference between the calculated and measured gas path parameters $\left(\mathbf{z}_{i \text { real }}-\mathbf{z}_{i \text { cal }}\right)$ is used by QPSO to evaluate the solutions. The objective function used in QPSO algorithm is defined as the following equation:

$$
\mathrm{OF}=\sqrt{\sum_{i=1}^{M} \frac{\left(\left(\mathbf{z}_{\text {ireal }}-\mathbf{z}_{i \mathrm{cal}}\right) / \mathbf{z}_{\text {ireal }}\right)^{2}}{M}} .
$$

Then the engine health status estimation can be viewed as a minimization problem. In (8) $M$ is the number of measurement parameters. If the difference $\left(\mathbf{z}_{i \text { real }}-\mathbf{z}_{i \text { cal }}\right)$ meets the accuracy requirements (the value of OF is smaller than $\sigma)$, then the algorithm stops iteration and outputs the best solution, where $\sigma$ is a very small number (in this study, $\sigma$ is set as 0.001). Else, QPSO generates new population and repeats the procedures.

Then, the obtained best solution (engine components parameters) is used to calculate the engine health status index (i.e., deterioration scaling factor). The engine health status index can be defined as follows:

$$
\begin{array}{r}
\mathrm{SF}_{\eta, \mathrm{cl}}=\frac{\eta_{\mathrm{cl}, d}}{\eta_{\mathrm{cl}, 0}} \\
\mathrm{SF}_{w, \mathrm{cl}}=\frac{w_{\mathrm{cl}, d}}{w_{\mathrm{cl}, 0}},
\end{array}
$$


where $\eta_{\mathrm{cl}, d}$ denotes the calculated deteriorated low-pressure compressor efficiency and $\eta_{\mathrm{cl}, 0}$ denotes the initial undeteriorated efficiency. $\mathrm{SF}_{\eta, \mathrm{cl}}$ denotes the engine health status index of low-pressure compressor efficiency. Likewise, $\mathrm{SF}_{w, \mathrm{cl}}$ stands for the engine health status index of low-pressure compressor gas flow rate and is calculated by the deteriorated and undeteriorated low-pressure compressor gas flow rate.

It should be noted that the components characteristic maps and thermodynamic engine performance model are used to calculate engine gas path parameters and there is not any linearization during the optimization procedures. This is quite different from traditional GPA methods which are based on linearized engine model. And this helps the QPSO method outperform GPA methods in estimation accuracy especially when the nonlinearity of the engine model is significant.

The detailed steps of QPSO-based engine health status estimation are described as follows.

Step 1. Initializing. First, generate a population of candidate solutions $\mathbf{x}, \mathbf{x}=\left\{x_{1}, x_{2}, \ldots, x_{N}\right\}$.

Step 2. Fitness Evaluation. Each particle in population is used to obtain components parameters $\pi_{\mathrm{cl}}, \pi_{\mathrm{ch}}, \Delta H_{\mathrm{tl}}$, and $\Delta H_{\text {th }}$ from components characteristics maps. Then these parameters are put into engine performance model and gas path parameter vector $\mathbf{z}_{i c a l}$ is obtained.

Step 3. Error Calculation. The calculated gas path parameter vector $\mathbf{z}_{i c a l}$ is compared with the real one $\mathbf{z}_{i \text { real }}$ and the error (objective function value) is obtained using (8). If the error is less than $\sigma$ (or the maximum number of iterations is reached), output the best solution $g B e s t$ and calculated engine health status indices; otherwise, go on to the next step.

Step 4. Update each particle's best previous position $p$ Best ${ }_{i}$ and the global best position $g$ Best.

Step 5. Calculate each particle's local attractor $\mathbf{P}_{i}$ and mean best position $m$ Best.

Step 6. Update particle's new position according to equation (6); return to Step 2.

\section{Experiments and Discussions}

4.1. Experiments Setting. In order to test the performance of the developed QPSO-based engine health estimation method, a certain type of turbine fan engine (for confidentiality reasons the engine type is omitted) performance model is used for the comparison of different estimation methods. The main components characteristic maps are provided by engines' manufacturer. This engine is a two-shaft turbine fan engine with a mixer and an afterburner. It is illustrated as in Figure 3. The numbers in the figure stand for the inlet or outlet of different components. For example, "3" stands for the outlet of high-pressure compressor or the inlet of main combustor and " 68 " stands for the outlet of afterburner or the inlet of nozzle (see Figure 3).
In this study, eight components parameters are selected. These parameters include flow rate and efficiency of compressors and turbines which are listed in the first two columns of Table 1. Accordingly eight gas path parameters are applied to obtain these eight components parameters. The symbols and units of the eight parameters are listed in Table 2.

The deterioration of the engine is simulated by implantation of the degradation in different components. In this study, four engine degradation cases are designed to compare the performance of these methods, as listed in Table 1. The first two cases have single component degradation in low-pressure compressor and low-pressure turbine separately. The third case has two degradations in low-pressure compressor and high-pressure compressor. In the fourth case, there are four degradations in all components simultaneously. The first two cases are designed to test the capability of each method in isolating and quantifying if the degradation happened only in one component, while the last two cases intend to test whether the method can quantify the degraded components when degradations happen in more than one component.

In order to test the adaptability of the proposed method, three different engine operating conditions represented by high and low rotational speeds are selected. They are as follows.

$$
\begin{aligned}
& \text { Condition 1: } N_{h}=95.89 \%, N_{l}=87.76 \% . \\
& \text { Condition 2: } N_{h}=93.79 \%, N_{l}=84.59 \% . \\
& \text { Condition 3: } N_{h}=91.22 \%, N_{l}=80.27 \% .
\end{aligned}
$$

Among these three engine operating conditions, the first condition is the closest to the engine design-point condition, while the other two are a little far away from the engine design-point condition.

When QPSO is applied, it first generates a population of candidate solutions $\mathbf{x}, \mathbf{x}=\left\{x_{1}, x_{2}, \ldots, x_{k}, \ldots, x_{N}\right\}$, where $x_{k}$ denotes a certain component parameter and has its own limits. As a particle is randomly initialized within the range of $[0,1], x_{k}$ is calculated as follows:

$$
x_{k}=\left(l_{k}^{\text {upper }}-l_{k}^{\text {lower }}\right) x_{k}^{0}+l_{k}^{\text {lower }},
$$

where $x_{k}^{0}$ is the $k$ th dimension of initialized particle $x^{0}$ and $l_{k}^{\text {upper }}, l_{k}^{\text {lower }}$ are the upper and lower bounds of the corresponding component parameter.

During iterations, if some $x_{i}$ is beyond the upper or lower limit, it is adjusted as follows:

$$
x_{k}= \begin{cases}l_{k}^{\text {upper }}-\varepsilon\left(l_{k}^{\text {upper }}-l_{k}^{\text {lower }}\right) N(0,1) & \text { if } x_{k} \geq l_{k}^{\text {upper }} \\ l_{k}^{\text {lower }}+\varepsilon\left(l_{k}^{\text {upper }}-l_{k}^{\text {lower }}\right) N(0,1) & \text { if } x_{k}<l_{k}^{\text {lower }},\end{cases}
$$

where $\varepsilon=0.01$ and $N(0,1)$ is a random number uniformly distributed in the interval $[0,1]$.

4.2. Parameter Setting of Different Methods. To evaluate the QPSO-based estimation method, it is compared with GAbased method, adaptive linear GPA method, and adaptive nonlinear GPA method. These four methods are applied to 


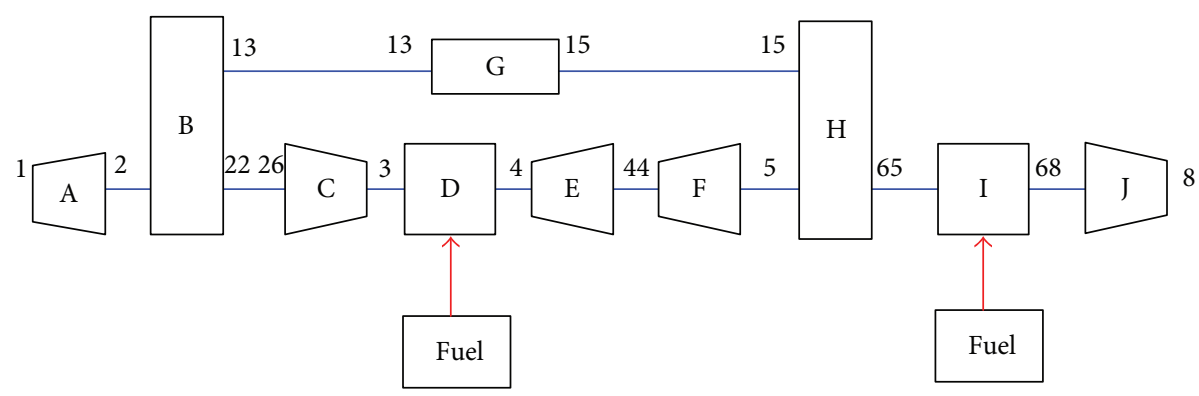
(A) Inlet
(B) Fan
(C) High-pressure compressor
(F) Low-pressure turbine
(C) High-pressure co
(D) Main combustor
(E) High-pressure turbine
(G) External duct
(H) Mixer
(I) Afterburner
(J) Nozzle

FIGURE 3: Schematic of studied turbine fan engine.

TABLE 1: Implanted components degradation of 4 test cases.

\begin{tabular}{|c|c|c|c|c|}
\hline \multirow{2}{*}{ Component health parameters } & \multicolumn{4}{|c|}{ Implanted degradation (\%) } \\
\hline & Case 1 & Case 2 & Case 3 & Case 4 \\
\hline \multicolumn{5}{|l|}{ Low pressure compressor } \\
\hline Efficiency $\eta_{\mathrm{cl}}$ & -1.0 & 0 & -1.0 & -1.0 \\
\hline Gas flow rate $w_{\mathrm{cl}}$ & -3.0 & 0 & -3.0 & -3.0 \\
\hline \multicolumn{5}{|l|}{ High pressure compressor } \\
\hline Efficiency $\eta_{\mathrm{ch}}$ & 0 & 0 & -1.0 & -1.0 \\
\hline Gas flow rate $w_{\mathrm{ch}}$ & 0 & 0 & -2.0 & -2.0 \\
\hline \multicolumn{5}{|l|}{ Low pressure turbine } \\
\hline Efficiency $\eta_{\mathrm{tl}}$ & 0 & -1.0 & 0 & -1.0 \\
\hline Gas flow rate $w_{\mathrm{tl}}$ & 0 & -3.0 & 0 & -3.0 \\
\hline \multicolumn{5}{|l|}{ High pressure turbine } \\
\hline Efficiency $\eta_{\text {th }}$ & 0 & 0 & 0 & -1.0 \\
\hline Gas flow rate $w_{\text {th }}$ & 0 & 0 & 0 & -2.0 \\
\hline
\end{tabular}

the four cases as listed in Table 1. In order to compare their adaptability, for each case, they are applied to three different engine operation conditions as listed in Section 4.1.

As GA and QPSO are random searching algorithms, the GA-based and QPSO-based methods may output different solutions even if the calculation is repeated with the same initial conditions. In order that these two methods provide reliable solutions, calculations with the same initial condition and parameter settings are repeated five times and the best result among the five results is selected as the output result.

According to the steps of QPSO-based engine health status estimation, if the objective function value is smaller than $\sigma$, the convergence of optimization process is declared. Also the maximum number of iterations needs to be predefined in case that the method is trapped into the endless loop. If the method does not obtain a solution which meets the accuracy requirements within 50 iterations, then it is forced to stop. In this study, the population size and the maximum number of iterations are set as in Table 3.

The contraction-expansion coefficient of QPSO is set to decrease from 1.0 to 0.5 linearly with the iterations. For GA algorithm, its parameters are set as follows: the probability of crossover is 0.5 and the probability of mutation increases linearly from 0.3 to 0.7 with the iterations as suggested in [4]. For GPA methods, the influence coefficient matrix $\mathbf{H}$ is obtained by the linearization of thermodynamic engine performance model.

\subsection{Discussions}

4.3.1. Comparison of the Accuracies of the Methods. Four cases from Table 1 are evaluated on 3 engine operating conditions by the IGPA (adaptive linear GPA), nGPA (adaptive nonlinear GPA), GA-based method, and QPSObased method separately and the detailed results are listed in Tables 4, 5, 6, and 7. Figures 4, 5, 6, and 7 are the mean relative error of the engine health status indices by the four algorithms on three conditions for case 1 to case 4 . In order to demonstrate the results directly, Table 4 lists the engine degradation status parameters instead of health status indices.

For case 1, only low-pressure compressor was implanted with two parameters' degradation. It can be concluded from Table 4 and Figure 4 that, on operating condition 1, all the 


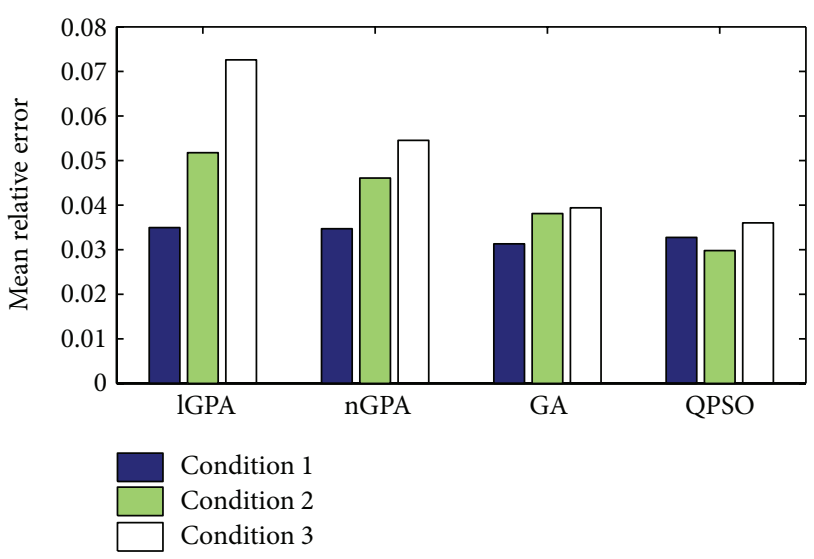

FIGURE 4: The mean relative errors of the engine health status indices by the four algorithms on three conditions for case 1 .

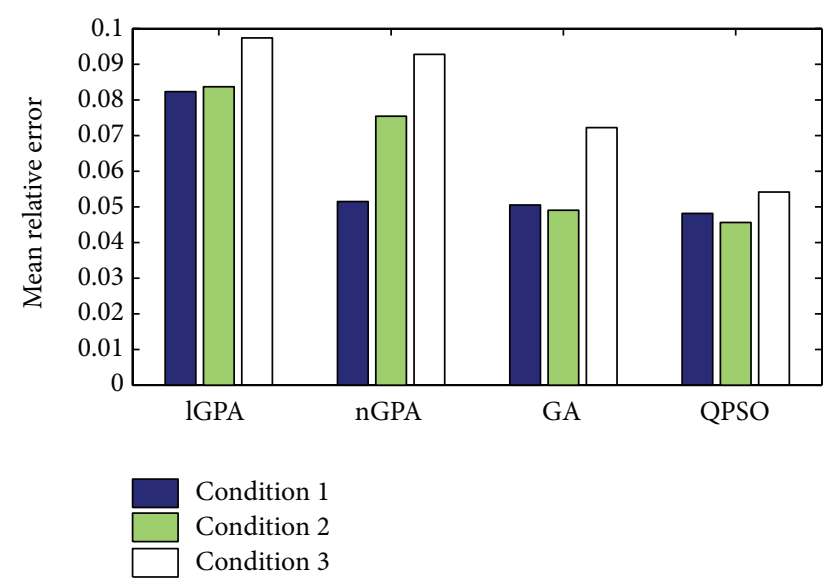

FIGURE 5: The mean relative errors of the engine health status indices by the four algorithms on three conditions for case 2 .

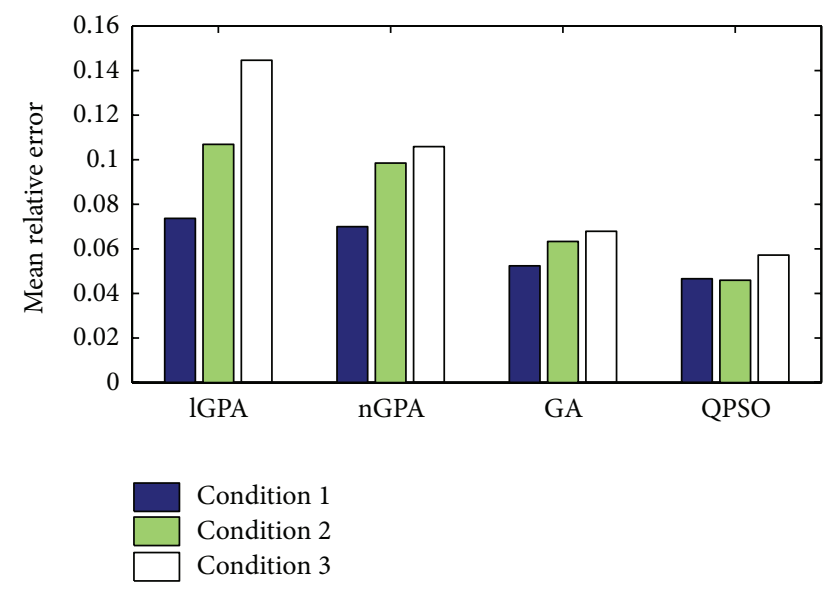

FIGURE 6: The mean relative errors of the engine health status indices by the four algorithms on three conditions for case 3 .
TABLE 2: Engine gas path instrumentation set.

\begin{tabular}{lcll}
\hline Num. & Symbol & Unit & Parameters \\
\hline 1 & $F$ & $\mathrm{kN}$ & Engine thrust \\
\hline 3 & $W_{\text {in }}$ & $\mathrm{kg} / \mathrm{s}$ & $\begin{array}{l}\text { Engine intake gas flow } \\
\text { rate }\end{array}$ \\
\hline 4 & $P_{t 3}$ & $\mathrm{MPa}$ & $\begin{array}{l}\text { Total pressure at } \\
\text { high-pressure } \\
\text { compressor exit }\end{array}$ \\
\hline 5 & $T_{t 3}$ & $\mathrm{~K}$ & $\begin{array}{l}\text { Total temperature at } \\
\text { high-pressure } \\
\text { compressor exit }\end{array}$ \\
\hline 6 & $P_{t 5}$ & $\mathrm{MPa}$ & $\begin{array}{l}\text { Total pressure at } \\
\text { low-pressure compressor } \\
\text { exit }\end{array}$ \\
\hline 7 & $T_{t 5}$ & $\mathrm{~K}$ & $\begin{array}{l}\text { Total temperature at } \\
\text { low-pressure compressor } \\
\text { exit }\end{array}$ \\
\hline 8 & $P_{t 68}$ & $\mathrm{MPa}$ & $\begin{array}{l}\text { Total pressure at } \\
\text { afterburner exit }\end{array}$ \\
\hline
\end{tabular}

TABLE 3: Engine gas path instrumentation set.

\begin{tabular}{lcccc}
\hline \multirow{2}{*}{ Parameters } & \multicolumn{4}{c}{ Value } \\
& GA & QPSO & lGPA & nGPA \\
\hline Population size & 50 & 50 & - & - \\
Number of iterations & 50 & 50 & 50 & 50 \\
\hline
\end{tabular}

four methods gave an engine health estimation with a high accuracy. The best result of the mean relative errors of component parameters is $3.13 \%$ obtained by GA-based method. And others' results are also satisfactory. On conditions 2 and 3, the mean relative errors obtained by lGPA and nGPA methods become larger while the results of GA and QPSO maintain the same level. Also similar conclusions can be made from Table 5 and Figure 5 of case 2 with one degraded component (low-pressure turbine).

For case 3 and case 4 where more than one component is implanted with degradations, the accuracies become lower than case 1 and case 2 with only one degraded component. But the GA- and QPSO-based methods can obtain relatively better results than the two GPA-based methods.

From the above discussions, conclusions can be reached that GA-based method and QPSO-based method obtain better results than the two GPA methods on off-design conditions, while for GPA-based methods, on conditions that are far away from the engine design point, the estimated accuracy becomes lower.

On the other hand, although the estimation accuracy decreases as the number of degraded components increases, GA- and QPSO-based methods perform better than the others in all cases, and the best results are obtained by QPSO-based method. This can be seen from Figure 8. The reason mainly lies in that GPA methods are based on the 
TABLE 4: Comparisons of the four methods on test case 1.

\begin{tabular}{lccccccccccccccc}
\hline $\begin{array}{l}\text { Engine } \\
\text { degradation }\end{array}$ & $\begin{array}{c}\text { Implanted } \\
\text { degradation (\%) }\end{array}$ & Con1 & Con2 & Con3 & Con1 & Con2 & Con3 & Con1 & Con2 & Con3 & Con1 & Con2 & Con3 \\
\hline $\mathrm{SF}_{\eta, \mathrm{cl}}-1$ & -1.0 & -1.033 & -0.955 & -1.088 & -1.036 & -1.047 & -1.063 & -1.022 & -0.968 & -0.967 & -1.032 & -0.959 & -1.025 \\
$\mathrm{SF}_{w, \mathrm{cl}}-1$ & -3.0 & -3.078 & -3.157 & -3.212 & -3.086 & -3.174 & -2.83 & -3.067 & -2.873 & -3.092 & -2.896 & -2.926 & -3.112 \\
$\mathrm{SF}_{\eta, \mathrm{ch}}-1$ & 0 & 0.041 & -0.038 & 0.072 & -0.041 & 0.054 & 0.047 & 0.032 & 0.039 & 0.048 & 0.027 & 0.035 & 0.038 \\
$\mathrm{SF}_{w, \mathrm{ch}}-1$ & 0 & -0.037 & -0.062 & 0.076 & -0.037 & 0.054 & -0.049 & 0.034 & 0.042 & -0.034 & -0.037 & 0.025 & 0.045 \\
$\mathrm{SF}_{\eta, \mathrm{tl}}-1$ & 0 & -0.025 & -0.051 & 0.056 & 0.028 & -0.035 & 0.051 & -0.041 & 0.023 & 0.043 & 0.030 & 0.032 & -0.035 \\
$\mathrm{SF}_{w, \mathrm{tl}}-1$ & 0 & 0.041 & -0.063 & 0.054 & 0.040 & 0.030 & 0.045 & 0.033 & -0.035 & 0.040 & 0.036 & 0.022 & 0.035 \\
$\mathrm{SF}_{\eta, \mathrm{th}}-1$ & 0 & 0.044 & 0.052 & -0.086 & 0.036 & 0.037 & -0.05 & 0.029 & 0.043 & -0.04 & -0.039 & 0.035 & 0.027 \\
$\mathrm{SF}_{w, \mathrm{th}}-1$ & 0 & -0.032 & -0.05 & -0.076 & 0.030 & 0.052 & -0.073 & -0.035 & 0.046 & -0.045 & 0.026 & 0.022 & 0.044 \\
\hline
\end{tabular}

TABLE 5: Comparisons of the four methods on test case 2.

\begin{tabular}{lccccccccccccccc}
\hline $\begin{array}{l}\text { Engine } \\
\text { degradation }\end{array}$ & $\begin{array}{c}\text { Implanted } \\
\text { degradation (\%) }\end{array}$ & Con1 & Con2 & Con3 & Con1 & Con2 & Con3 & Con1 & Con2 & Con3 & Con1 & Con2 & Con3 \\
\hline $\mathrm{SF}_{\eta, \mathrm{cl}}-1$ & 0 & -0.092 & -0.067 & 0.094 & 0.060 & 0.090 & -0.077 & 0.038 & -0.064 & 0.085 & -0.043 & -0.049 & 0.059 \\
$\mathrm{SF}_{w, \mathrm{cl}}-1$ & 0 & 0.070 & 0.102 & 0.126 & -0.061 & 0.090 & -0.105 & 0.041 & 0.041 & 0.055 & -0.04 & -0.056 & 0.063 \\
$\mathrm{SF}_{\eta, \mathrm{ch}}-1$ & 0 & -0.086 & 0.082 & 0.095 & -0.064 & 0.093 & -0.113 & 0.058 & 0.049 & 0.087 & 0.041 & -0.038 & 0.065 \\
$\mathrm{SF}_{w, \mathrm{ch}}-1$ & 0 & -0.084 & 0.113 & 0.085 & 0.039 & 0.055 & 0.077 & -0.064 & 0.047 & 0.072 & -0.037 & 0.044 & -0.07 \\
$\mathrm{SF}_{\eta, \mathrm{tl}}-1$ & -1.0 & -1.088 & -0.93 & -1.092 & -1.044 & -1.077 & -1.066 & -0.942 & -1.043 & -1.083 & -0.941 & -0.97 & -0.964 \\
$\mathrm{SF}_{w, \mathrm{tl}}-1$ & -3.0 & -3.258 & -3.197 & -2.733 & -3.17 & -3.225 & -3.377 & -3.146 & -3.213 & -3.135 & -2.838 & -2.884 & -2.844 \\
$\mathrm{SF}_{\eta, \mathrm{th}}-1$ & 0 & -0.064 & 0.071 & -0.07 & 0.045 & 0.052 & -0.098 & 0.035 & -0.039 & -0.064 & 0.046 & 0.053 & 0.045 \\
$\mathrm{SF}_{w, \mathrm{th}}-1$ & 0 & 0.087 & -0.098 & -0.127 & -0.041 & -0.069 & 0.079 & -0.06 & 0.037 & 0.084 & 0.063 & 0.054 & 0.041 \\
\hline
\end{tabular}

TABLE 6: Comparisons of the four methods on test case 3.

\begin{tabular}{lcccccccccccccc}
\hline $\begin{array}{l}\text { Engine } \\
\text { degradation }\end{array}$ & $\begin{array}{c}\text { Implanted } \\
\text { degradation (\%) }\end{array}$ & Con1 & Con2 & Con3 & Con1 & Con2 & Con3 & Con1 & Con2 & Con3 & Con1 & Con2 & Con3 \\
\hline $\mathrm{SF}_{\eta, \mathrm{cl}}{ }^{-1}$ & -1.0 & -1.074 & -1.134 & -0.833 & -0.934 & -0.931 & -0.908 & -1.059 & -1.065 & -1.055 & -1.038 & -1.035 & -0.948 \\
$\mathrm{SF}_{w, \mathrm{cl}}-1$ & -3.0 & -2.819 & -3.33 & -3.373 & -2.845 & -2.637 & -2.551 & -3.128 & -2.853 & -3.265 & -3.175 & -3.141 & -2.817 \\
$\mathrm{SF}_{\eta, \mathrm{ch}}-1$ & -1.0 & -0.91 & -1.114 & -1.14 & -1.058 & -1.125 & -1.092 & -0.934 & -1.051 & -1.069 & -1.051 & -1.045 & -0.956 \\
$\mathrm{SF}_{w, \mathrm{ch}}-1$ & -2.0 & -2.153 & -2.191 & -2.308 & -2.14 & -1.861 & -2.187 & -1.912 & -2.094 & -2.11 & -2.064 & -2.127 & -2.088 \\
$\mathrm{SF}_{\eta, \mathrm{tl}}-1$ & 0 & -0.059 & -0.124 & 0.164 & -0.086 & 0.098 & 0.148 & 0.039 & 0.077 & 0.057 & 0.055 & 0.035 & -0.074 \\
$\mathrm{SF}_{w, \mathrm{tl}}-1$ & 0 & -0.106 & 0.101 & -0.137 & 0.090 & 0.084 & 0.087 & 0.046 & 0.065 & 0.053 & -0.054 & 0.062 & 0.079 \\
$\mathrm{SF}_{\eta, \mathrm{th}^{-1}}$ & 0 & -0.066 & -0.077 & 0.133 & 0.086 & -0.096 & 0.090 & -0.064 & 0.079 & 0.084 & -0.044 & 0.041 & 0.059 \\
$\mathrm{SF}_{w, \mathrm{th}}-1$ & 0 & 0.057 & -0.1 & -0.137 & -0.051 & 0.124 & -0.093 & 0.057 & 0.071 & 0.080 & -0.04 & -0.036 & -0.044 \\
\hline
\end{tabular}

TABLE 7: Comparisons of the four methods on test case 4.

\begin{tabular}{lcccccccccccccc}
\hline $\begin{array}{l}\text { Engine } \\
\text { degradation }\end{array}$ & $\begin{array}{c}\text { Implanted } \\
\text { degradation (\%) }\end{array}$ & Con1 & Con2 & Con3 & Con1 & Con2 & Con3 & Con1 & Con2 & Con3 & Con1 & Con2 & Con3 \\
\hline $\mathrm{SF}_{\eta, \mathrm{cl}}-1$ & -1.0 & -1.11 & -0.916 & -1.126 & -1.093 & -1.114 & -1.178 & -1.047 & -1.085 & -1.075 & -1.062 & -1.081 & -0.954 \\
$\mathrm{SF}_{w, \mathrm{cl}}-1$ & -3.0 & -3.333 & -3.249 & -3.57 & -2.739 & -3.281 & -2.691 & -2.773 & -3.148 & -2.676 & -2.885 & -3.212 & -2.779 \\
$\mathrm{SF}_{\eta, \mathrm{ch}}-1$ & -1.0 & -0.913 & -1.092 & -1.221 & -1.066 & -0.903 & -0.848 & -0.93 & -0.945 & -1.078 & -1.048 & -1.07 & -0.924 \\
$\mathrm{SF}_{w, \mathrm{ch}}-1$ & -2.0 & -2.138 & -2.266 & -1.767 & -2.14 & -2.203 & -1.636 & -1.899 & -2.129 & -2.148 & -1.886 & -2.113 & -2.099 \\
$\mathrm{SF}_{\eta, \mathrm{tl}}-1$ & -1.0 & 0.064 & 0.107 & 0.168 & -0.1 & -0.124 & -0.188 & 0.049 & 0.052 & -0.057 & 0.067 & -0.076 & -0.062 \\
$\mathrm{SF}_{w, \mathrm{tl}}-1$ & -3.0 & 0.106 & 0.146 & -0.124 & 0.076 & -0.145 & 0.152 & -0.062 & 0.052 & -0.075 & 0.052 & 0.078 & -0.08 \\
$\mathrm{SF}_{\eta, \mathrm{th}}-1$ & -1.0 & 0.084 & -0.105 & -0.133 & -0.06 & 0.105 & -0.097 & 0.072 & 0.070 & 0.079 & 0.069 & -0.043 & 0.046 \\
$\mathrm{SF}_{w, \mathrm{th}}-1$ & -2.0 & 0.061 & 0.091 & 0.147 & 0.063 & 0.119 & 0.097 & 0.040 & 0.049 & 0.076 & 0.047 & -0.062 & 0.086 \\
\hline
\end{tabular}


TABLE 8: Comparisons of computing speed of the four methods on test case 2 .

\begin{tabular}{lcccc}
\hline $\begin{array}{l}\text { Computation times } \\
\text { (second) }\end{array}$ & IGPA & nGPA & GA & QPSO \\
\hline Max. & 0.29 & 4.1 & 1073 & 1062 \\
Min. & 0.16 & 2.7 & 709 & 848 \\
Mean & 0.19 & 3.1 & 849 & 925 \\
\hline
\end{tabular}

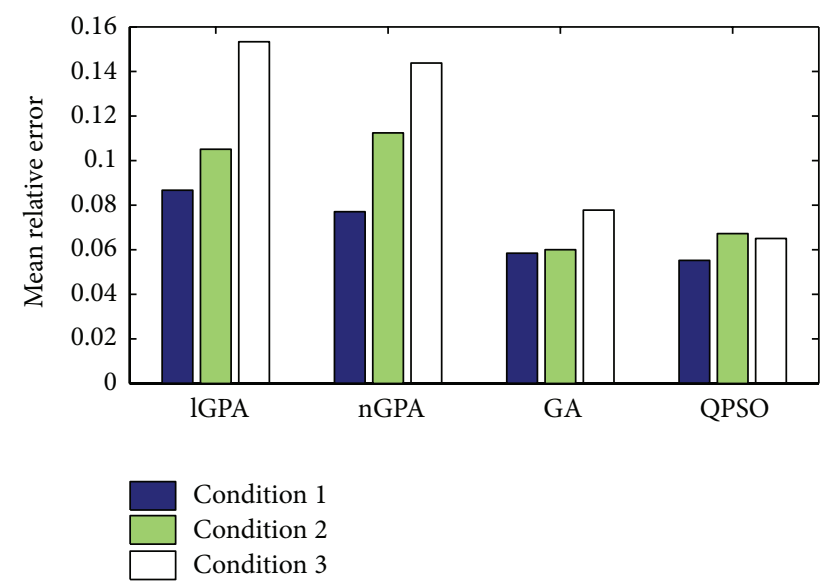

FIGURE 7: The mean relative errors of the engine health status indices by the four algorithms on three conditions for case 4 .

linearization of thermodynamic engine model while the GAbased method and QPSO-based method solve the problem directly and do not change the nonlinear nature of the engine operation, which helps them to attain higher estimation accuracies especially when the nonlinearity of the engine model is significant.

4.3.2. Comparisons of the Computation Speeds of the Four Methods. In this study all algorithms are run with an Intel Pentium IV dual core $2.5 \mathrm{GHz}$ PC at 1.99 GB RAM under a Microsoft Windows XP environment. Table 8 shows the comparisons of the computation speeds of these methods on case 2. The maximum and minimum computation times among the five independent runs and their mean values are listed in the table. Because the natures of GPA methods and GA-based method and QPSO-based method are very different, their computing speeds have big difference. GA-based method and QPSO-based method are much more time-consuming than GPA methods. For lGPA, the computational time is only a fraction of a second. It takes 3.1 seconds for nGPA to get a solution. However, for GA-based method and QPSO-based method, it takes them more than 14 minutes to obtain the solutions averagely. This makes it impossible for GA- and QPSO-based methods to be applied to the real-time engine performance analysis. But they are suitable to be applied to off-line engine health and performance estimation.

4.3.3. Comparison of GA-Based Method and QPSO-Based Method. As is concluded that GA-based method and QPSObased method perform better than the two GPA methods,

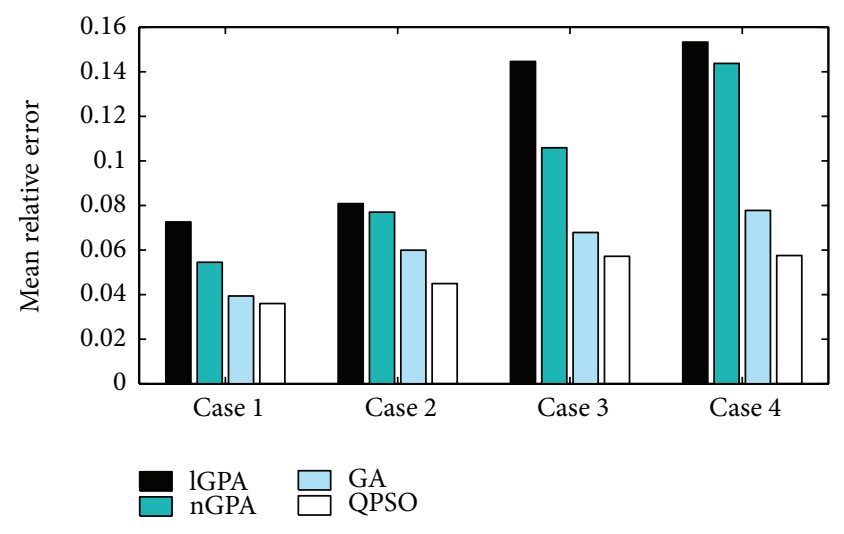

FIGURE 8: The mean relative errors of component parameters by the four methods in different cases.

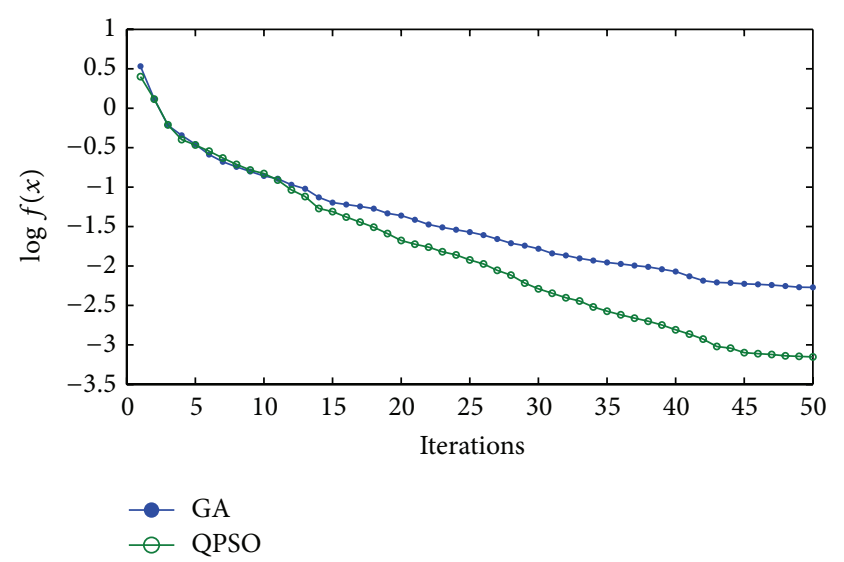

FIGURE 9: The average logarithm values of the objective function by the two methods during iterations.

in this section, these two methods are compared and it is decided which one is a more appropriate method for engine health status estimation.

The most important performance for these two population-based random searching algorithms is convergence performance or the effectiveness of obtaining the problem's solutions. So the algorithms' convergence performances are compared in different ways.

Figure 9 shows the average objective function logarithm values of 10 separate runs calculated by the two methods for the third condition of case 4 . It can be seen from the figure that the QPSO-based method obtains better convergence performance than GA-based method during iterations and the logarithm value of the objective function reaches -3.24 , while it is -2.68 for GA-based method after 50 iterations. In that case, GA-based method failed to attain the accuracy requirement $(\sigma=0.001, \log f(x)=-3)$.

Figure 10 shows the box plot chart of two methods' logarithm values of the objective functions at the end of iterations in 10 separate runs (the maximum iteration time is 50 for each run). It can be seen from the figure that, on all the three conditions, QPSO-based method has better result than GA-based method. The average logarithm values 

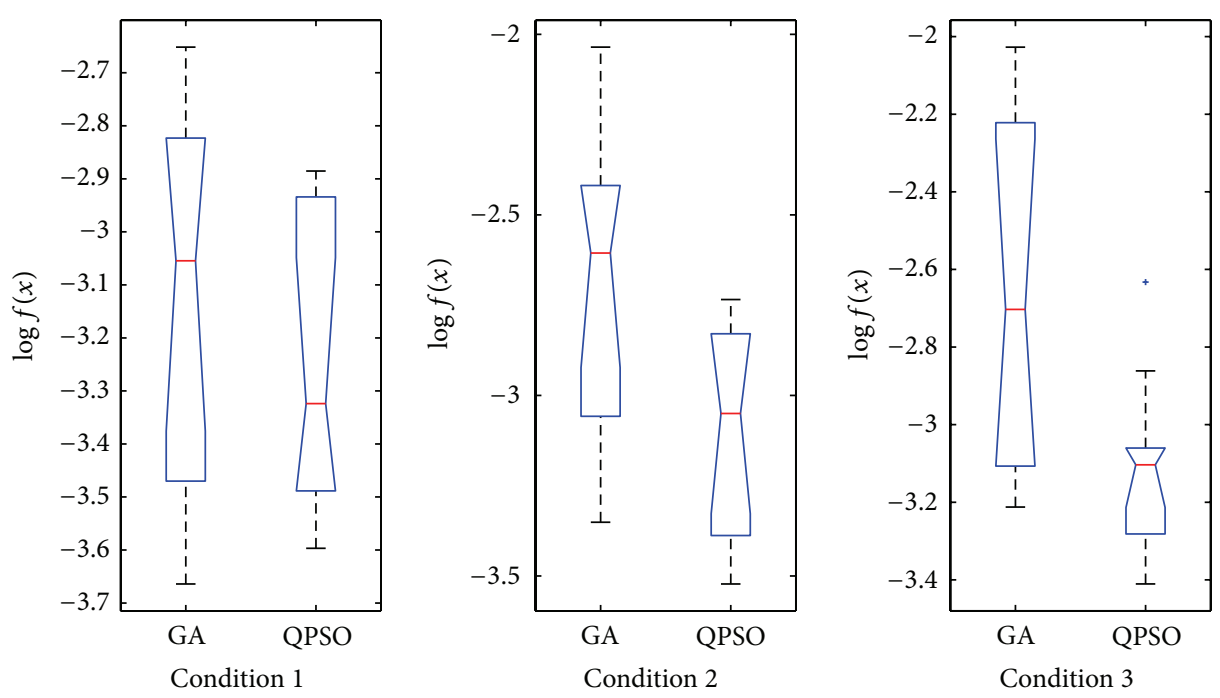

FIgURE 10: The box plot chart of two methods' results on three conditions for case 4 .

of the objective function are all less than -3 , while for GAbased method the logarithm values of the objective function on condition 2 and condition 3 are larger than -3 , which means that GA failed to meet the accuracy requirement in 50 iterations on these two conditions. In order to obtain solutions with higher accuracy, more iteration times are needed for GA-based method. On the contrary it takes less iteration times for QPSO-based method to obtain solutions with the same accuracy level.

\section{Conclusions}

In this study, a novel gas turbine engine health status estimation method has been introduced and described in detail. The method employs quantum-behaved particle swarm optimization algorithm as an optimization tool to estimate degraded engine health status. In order to test the performance of the developed method, it is applied to health status estimation of a gas turbine fan engine and is compared with the other three methods: adaptive linear gas path analysis method, adaptive nonlinear gas path analysis method, and GA-based method. Engine health status estimation is carried out with different sets of implanted component degradations on three different conditions.

The results show that the developed method is better than adaptive linear and nonlinear GPA methods in accuracy performance at off-design operating points, outperforms GA-based method in convergence speed, and avoids the premature convergence problem. This is mainly because the quantum mechanics helps the particles to search more effectively in search space. Although the method is slower in computation speed than GPA methods and is not suitable for online applications, the QPSO-based method is proven to be one of the best tools for off-line engine health status estimation.

It should be pointed out that the study is carried out on condition that the number of gas path measurement parameters equals the component performance parameters. But sometimes the number of measurement parameters may be less than the to-be-optimized parameters; in that case, the application of the developed method could be a future research topic.

\section{Conflict of Interests}

The authors declare that there is no conflict of interests regarding the publication of this paper.

\section{References}

[1] L. A. Urban, Gas Path Analysis Applied to Turbine Engine Condition Monitoring, AIAA-72-1082.

[2] B. A. Roth, D. N. Mavris, and D. L. Doel, "Estimation of turbofan engine performance model accuracy and confidence bounds," in Proceedings of the 16th International Symposium on Air Breathing Engines, ISABE Paper no. 2003-1208, 2003.

[3] B. R. Roth, D. Mavris, D. L. Doel, and D. Beeson, "Highaccuracy matching of engine performance models to test data," ASME Paper No. GT 2003-38784.

[4] Y. G. Li and P. Pilidis, "GA-based design-point performance adaptation and its comparison with ICM-based approach," Applied Energy, vol. 87, no. 1, pp. 340-348, 2010.

[5] Y. G. Li, "Gas turbine performance and health status estimation using adaptive gas path analysis," Journal of Engineering for Gas Turbines and Power, vol. 132, no. 4, Article ID 041701, pp. 11-19, 2010.

[6] H. S. Tan, "Fourier neural networks and generalized single hidden layer networks in aircraft engine fault diagnostics," Journal of Engineering for Gas Turbines and Power, vol. 128, no. 4, pp. 773-782, 2006.

[7] C. Romessis and K. Mathioudakis, "Bayesian network approach for gas path fault diagnosis," Journal of Engineering for Gas Turbines and Power, vol. 128, no. 1, pp. 64-72, 2006.

[8] D. Martis, "Fuzzy logic estimation applied to Newton methods for gas turbines," Journal of Engineering for Gas Turbines and Power, vol. 129, no. 1, pp. 88-96, 2007. 
[9] R. W. Eustace, "A real-world application of fuzzy logic and influence coefficients for gas turbine performance diagnostics," Journal of Engineering for Gas Turbines and Power, vol. 130, no. 6, Article ID 061601, 9 pages, 2008.

[10] J. Kennedy and R. Eberhart, "Particle swarm optimization," in Proceedings of the IEEE International Conference on Neural Networks, pp. 1942-1948, Perth, Australia, December 1995.

[11] C. Chang-Yi and Y. Yong-Chun, "Solving of nonlinear equations based on PSO algorithm," Computer Application and Software, no. 5, pp. 137-139, 2006.

[12] Z. Miao, S. Xie, Y. Wu, L. Zhu, L. Wang, and C. Chen, "Aeroengine state variable modeling based on the improved particle swarm optimization," Journal of Propulsion Technology, vol. 33, no. 1, pp. 73-77, 2012.

[13] A. H. Gandomi, G. J. Yun, X.-S. Yang, and S. Talatahari, "Chaosenhanced accelerated particle swarm optimization," Communications in Nonlinear Science and Numerical Simulation, vol. 18, no. 2, pp. 327-340, 2013.

[14] F. van den Bergh and A. P. Engelbrecht, "A study of particle swarm optimization particle trajectories," Information Sciences, vol. 176, no. 8, pp. 937-971, 2006.

[15] J. Sun, C.-H. Lai, W.-B. Xu, Y. Ding, and Z. Chai, "A modified quantum-behaved particle swarm optimization," in Proceedings of the 7th International Conference on Computational Science (ICCS '07), pp. 294-301, Beijing, China, May 2007.

[16] D. Chen, J. Wang, F. Zou, W. Hou, and C. Zhao, "An improved group search optimizer with operation of quantum-behaved swarm and its application," Applied Soft Computing Journal, vol. 12, no. 2, pp. 712-725, 2012.

[17] J. Sun, W. Fang, V. Palade, X. Wu, and W. Xu, "Quantumbehaved particle swarm optimization with Gaussian distributed local attractor point," Applied Mathematics and Computation, vol. 218, no. 7, pp. 3763-3775, 2011.

[18] P. Philip and P. Fletcher, Gas Turbine Performance Second Edition, Blackwell, Oxford, UK, 2004. 


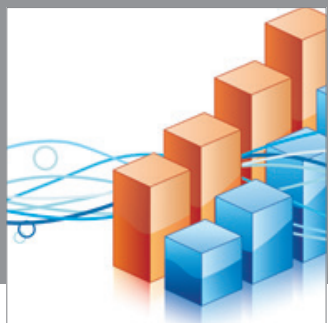

Advances in

Operations Research

mansans

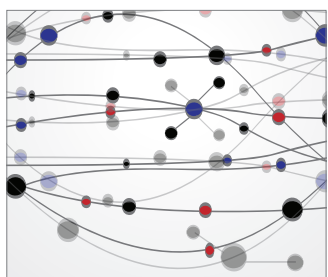

The Scientific World Journal
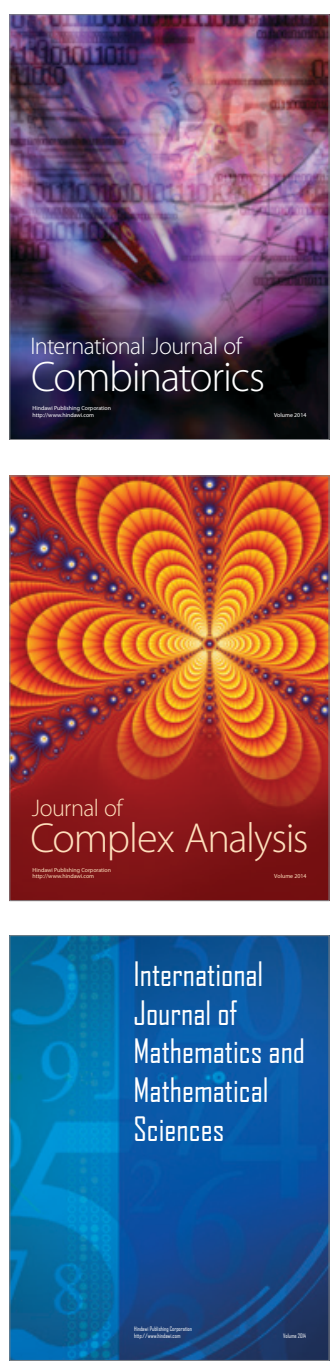
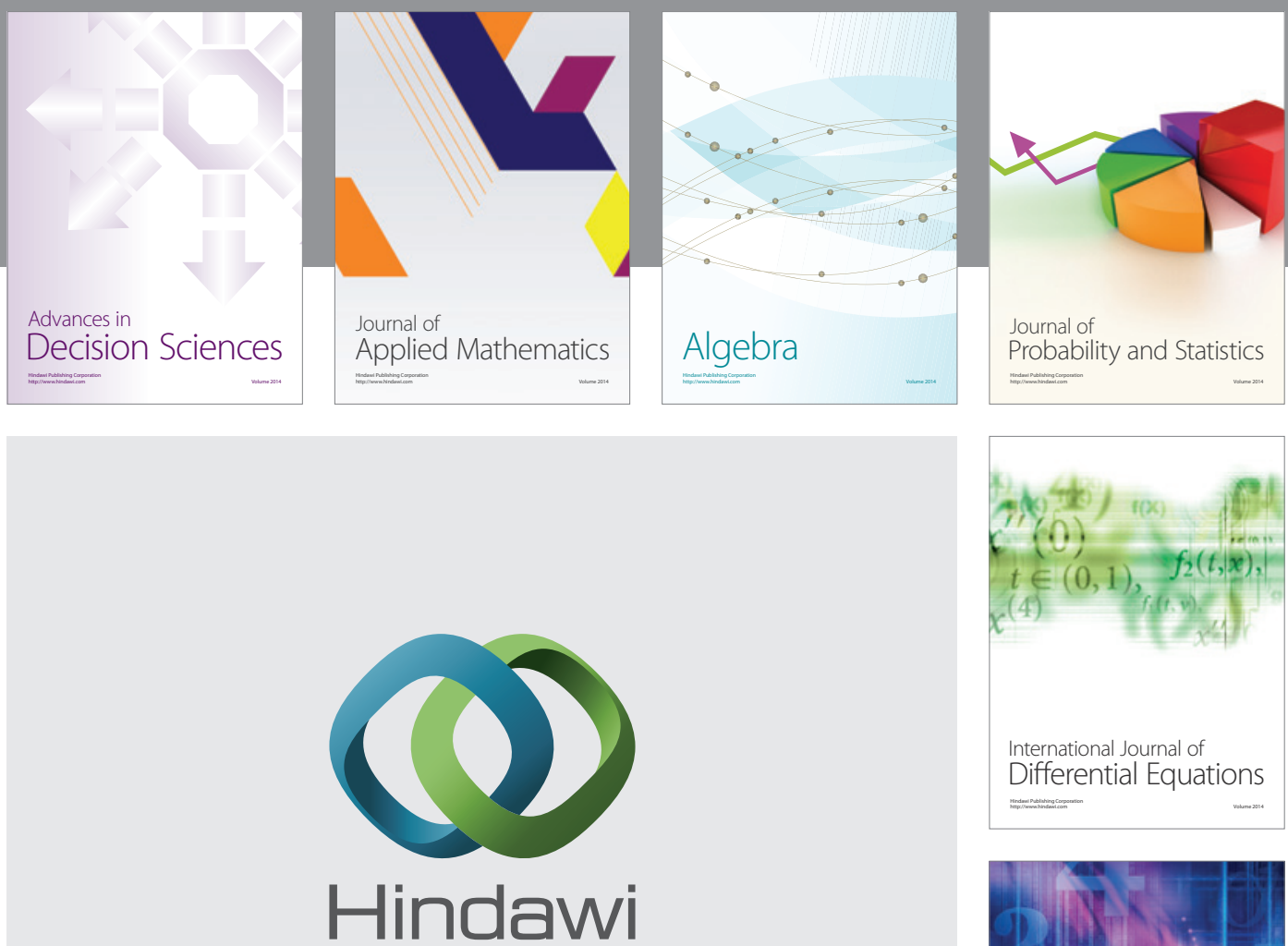

Submit your manuscripts at http://www.hindawi.com
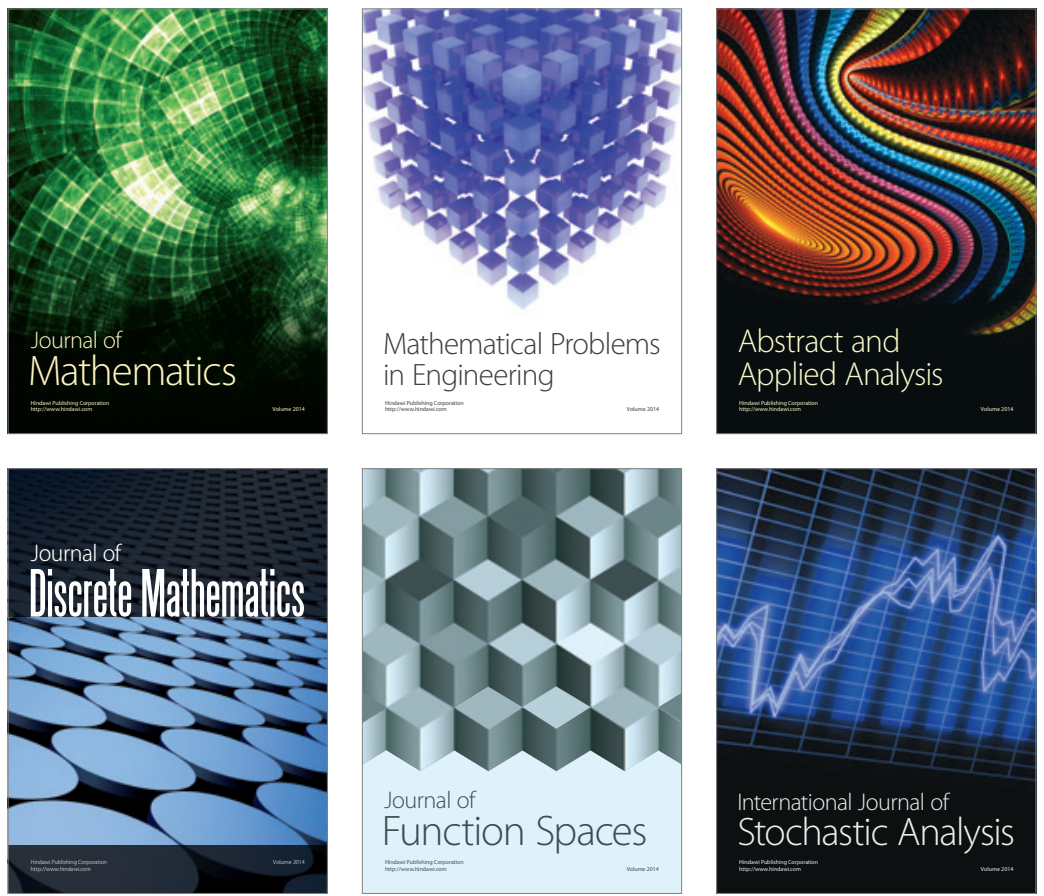

Journal of

Function Spaces

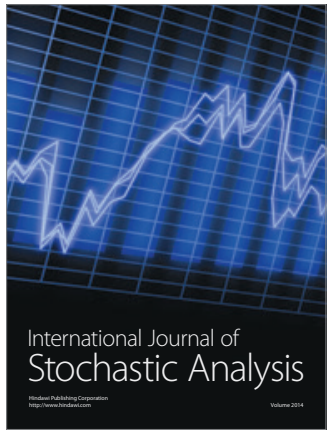

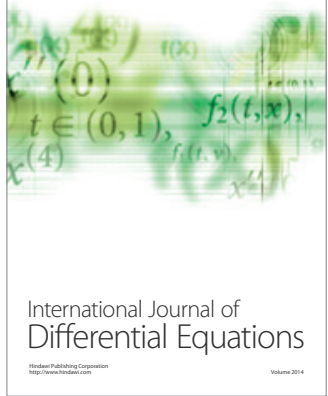
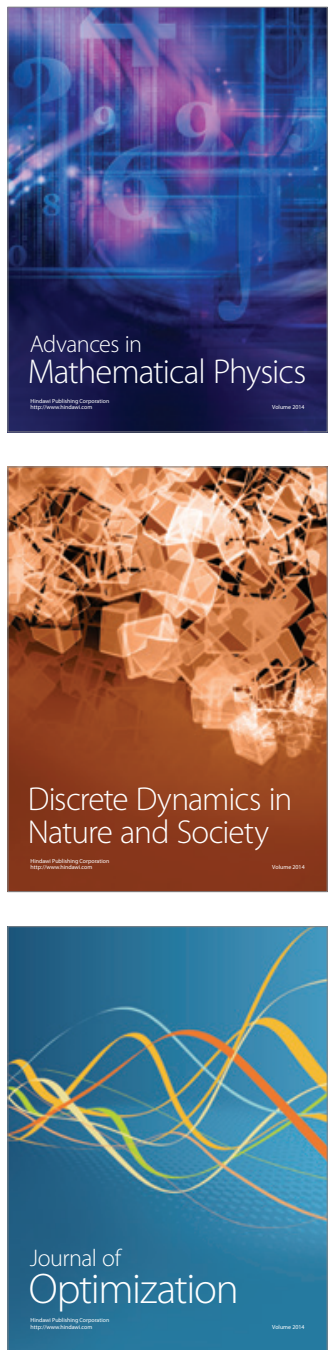